\title{
Optimization for the Storage Management and Job Scheduling Based on Expert System
}

\author{
Shuilong Zou ${ }^{1, a^{*}}$ and Ming $\mathrm{Hu}^{1, \mathrm{~b}}$ \\ ${ }^{1}$ Nanchang Institute of Science and Technology, Nanchang, 330108, China \\ a752782167@qq.com, b7752379@qq.com \\ *The Corresponding author
}

Keywords: Auto Control; Optimal; Expert System; Automated Storage; Stacker Crane

\begin{abstract}
In order to improve the efficiency of automated storage and retrieval system, expert systems were applied in the optimization of storage management and job scheduling of automated storage and retrieval system. By analyzing the experience of experts in the field, a knowledge base based on production rules was established. The expressions of knowledge are divided into three categories: storage allocation rules, tasks to sort rules and other rules. The application of forward inference engine designed the flow of reasoning, by which a more rational allocation of storage and quicker job scheduling were realized. Experimental results show that in the automated storage and retrieval system, applying expert system in the cargo space management and job scheduling optimization can effectively improve the operation efficiency of automated storage and retrieval system.
\end{abstract}

\section{Introduction}

Automated storage and retrieval system is a shelf storage system which using computer to control and manage for access operations implemented by automatic transport equipment. Cargo space management and job scheduling is the primary mean to improve the efficiency of automated storage and retrieval system. Traditional automated storage and retrieval system arranges the job order generally on the basis of the principle of priority in the process of job scheduling, and manually set job with a specific priority for the auxiliary. Which can generally meet users' normal requirements However, with increasing demands on the logistics efficiency and energy saving, the requirements for the operation efficiency of the automated storage and retrieval system are becoming higher and higher. Because the traditional method had been unable to meet people's requirements, the optimal scheduling problem of location assignment operation has been the industry's attention and carried out in-depth research which using a lot of methods such as simulated annealing, genetic algorithms, neural networks, Petri nets, etc. Simulated annealing algorithm is a kind of generalized random search algorithm, which can find the global optimal solution as far as possible. However, in order to ensure the better solution, the algorithm often takes slow cooling, multi-sampling, etc, resulting in a long run of the algorithm, which is the biggest drawback of simulated annealing. Genetic algorithm is a kind of parameter search algorithm based on natural selection and natural genetic mechanism. The choice of operator, such as selection, crossover and mutation, is easy to affect the quality of solution.

\section{The Cargo Space Management and Job Scheduling based on Expert System.}

Expert system is a kind of intelligent program system which integrates the experience and expertise of experts in the application domain, and then simulates the thinking process of human experts to solve the problem. Expert system is composed of 6 parts generally: man-machine interface, knowledge acquisition, knowledge base, reasoning machine, comprehensive database and interpretation system, in which knowledge base and reasoning machine are two significant components of expert system. The knowledge base stores the expertise about related field from the 
domain experts. The structure of automated storage and retrieval system about cargo space management and job scheduling based on the expert system is shown in Fig 1.

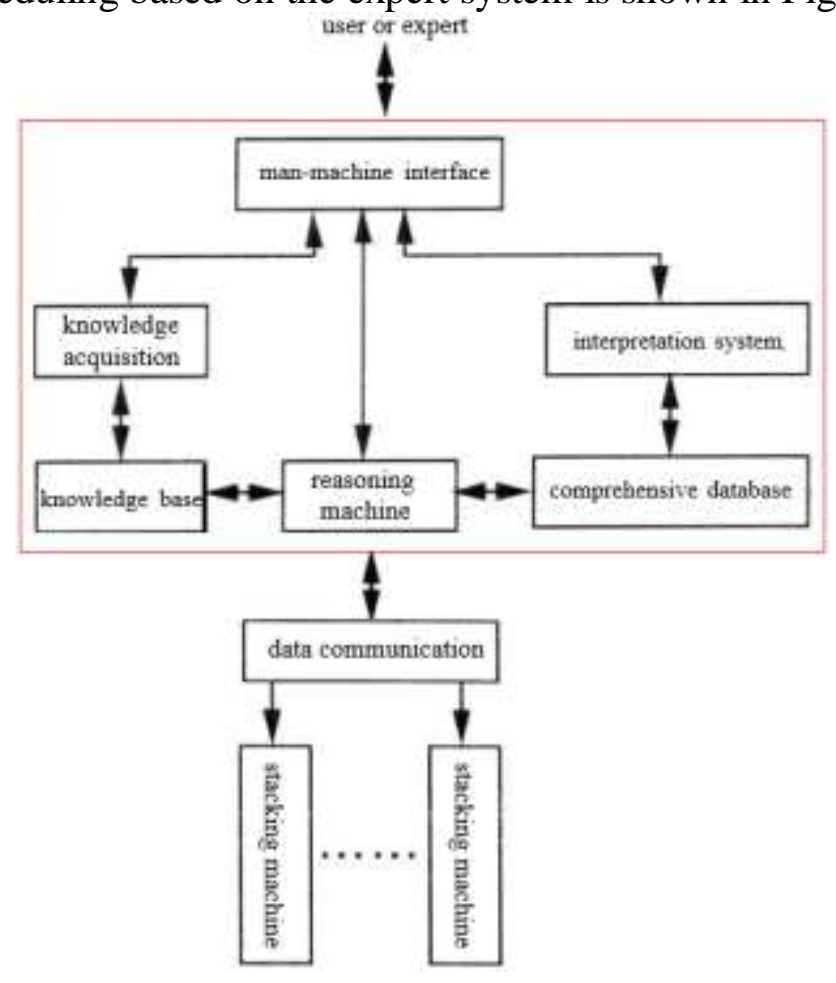

Fig 1. The structure of automated storage based on expert system

\section{The Knowledge Base of Automated Storage and Retrieval System}

Goods Position Management. In general, the allocation of goods should meet the following principles to ensure the efficiency of in-out storage:

1) Shelf load evenly by laying the heavy under the light and storing goods separately.

2) Speed up the turnover, first in first out. When the same kinds of goods out of the storage, we will follow the principle of first in first out to speed up the turnover of goods and effectively avoid the damage of corrosion, deformation, deterioration caused by long-term backlog of goods and the loss from other problems.

3)The principle of uniform distribution. In the centralized storage, the same kind of goods will be distributed to different roadway to enter the storage. It can improve the entry speed and prevent the roadway congestion that affect out-in storage operation.

4) The principle of the nearest. In order to ensure rapid response to the storage request, the goods will be placed nearby the entrance of storage platform.

5) Partition management. The shelf unit is managed according to the frequency of in-out storage, as shown in Fig 2. The area A (near the entrance of storage platform) is the area with the highest frequency of out-in storage. The area $\mathrm{B}$ is the normal storage region. The area $\mathrm{C}$ is the long-time storage region. The area $\mathrm{D}$ is the common region. 


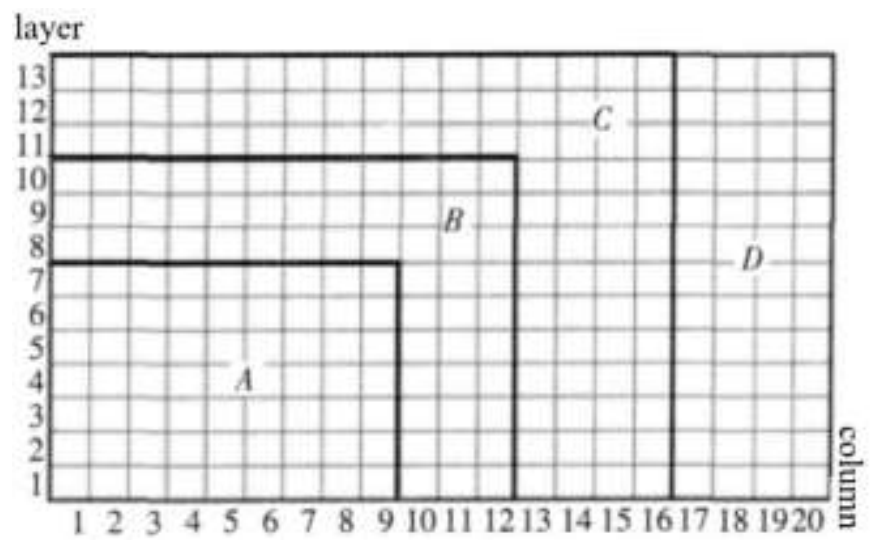

Fig 2. Shelf cargo area map grid cell

Job Scheduling for Out-in of Storage. Reasonable scheduling is one of the most effective means to improve the efficiency of automated storage and retrieval system, the main scheduling principles as follows :

1) The out of storage task is implemented firstly. When there are several tasks at the same time about out of the storage, we will implement the most urgent task firstly.

2) We implement the mission of entering storage until the implementation of the entering storage task does not affect other exit storage tasks being completed on time.

3) If the stacking machine of the entering task is faulty, the task will be stopped.

4) We should follow the principle of priority in job scheduling to ensure that first response to the highest priority task, namely the highest operational tasks of real-time requirements.

The Knowledge Representation of Automated Storage and Retrieval System. There are many ways to express knowledge in expert system, such as logical representation, semantic network representation, etc. According to the characteristics of automated storage and retrieval system, we adopt the generative rule representation in this paper which is most widely used. In the light of the experience about cargo space management and job scheduling optimization of the field expert, the knowledge in the knowledge base is composed of the distribution rules, task scheduling rules and other rules. Part of the rules are as follows (where R represents the rule):

1)Cargo space allocation rules

R1:if the goods are stored in the storage then the frequency $\mathrm{F}=\mathrm{N}+1(\mathrm{~N}$ is the number of enter stored within a month);

R2: if the frequency F>15 times / month and the goods entered the storage then the goods are put in the A part of storage;

R3:if the frequency of 5 times / month $\leq \mathrm{F} \leq 15$ times / month and goods entered storage then the goods are putted into the part B of storage;

2)Task sorting rules

$\mathrm{R} 1$ : If the task is in turn and there is no time requirement, then according to the order of the service;

R2: if there are numbers in-storage tasks and shortest time requires then path optimization;

R3: if there are emergency in-storage task then first execute the task;

R4: if there are the same frequency in-storage tasks then first execute the task that less in-storage number;

3) Others rules

$\mathrm{R} 1$ : if the state of the stacker is idle then the stacker number is obtained;

$\mathrm{R} 2$ : if the stacker is numbered 1 and the stacker is idle then the 1 \# stacker is issued instruction;

$\mathrm{R} 3$ : if the stacker numbered is 2 and the stacker is free then the 2 \# stacker is issued instruction; 


\section{The Inference Machine for Automated Storage and Retrieval System}

The inference machine is an important part of the expert system. Whose main task is to search for the corresponding knowledge according to current state to to solve the problem. There are three main methods: forward reasoning, reverse reasoning and forward-backward hybrid reasoning. In this paper,we adopt the forward reasoning algorithm which is used more commonly. The main work includes:

1) According to a certain strategy, the rules are chosen from the knowledge base to match with the facts in the comprehensive database. The corresponding rules can be used when the prerequisites of the rule in the comprehensive database are consistent with the known facts or it approximately consistent with the fact and meet the prerequisites. Otherwise the inference machine will match with the next rule in the knowledge base.

2) If there are more than one rule which be matched successful, the reasoning agency will call the corresponding policy to digest to select one be executed.

\section{Experiment Results}

The configuration of automated storage and retrieval system which in CNC machine factory of an aviation stock company is as follows: There are four rows of shelves, with a total of 20 rows of 13 storey; unit cargo grid length is $1.67 \mathrm{~m}$, width $0.8 \mathrm{~m}$, height $0.7 \mathrm{~m}$. Horizontal running speed of stacking machine is $100 \mathrm{~m} / \mathrm{min}$ and acceleration is $0.3 \mathrm{~m} / \mathrm{s} 2$; the vertical running speed of stacking machine is $25 \mathrm{~m} / \mathrm{min}$ and acceleration is $0.2 \mathrm{~m} / \mathrm{s} 2$; the speed of stacker fork is $20 \mathrm{~m} / \mathrm{min}$ and acceleration is $0.2 \mathrm{~m} / \mathrm{s} 2$. For the above three-dimensional storage, the program would be made with Delphi7.0 and SQL Serv-er2000 database to implement expert system. 20 goods are selected for out-in storage operations experiment and repeat the operation 10 times, the data shown in Table 1. It can be seen from Table 1 that the time required for storage in a random storage operation is large and the average storage time is $1647.6 \mathrm{~s}$. The average time of storage operation using neural network algorithm is $1607.4 \mathrm{~s}$. The average storage time of the expert system for storage operation is $1575.2 \mathrm{~s}$. Compared with the random network operation, the time saving is $72.4 \mathrm{~s}$, compared with the neural network algorithm, the time saving is $32.2 \mathrm{~s}$, and the variation range is small.

Tab.1 Experimental data table

\begin{tabular}{cccc}
\hline oder number & sheme $1(\mathrm{~s})$ & sheme $2(\mathrm{~s})$ & sheme $3(\mathrm{~s})$ \\
\hline 1 & 1640 & 1613 & 1588 \\
2 & 1568 & 1590 & 1582 \\
3 & 1440 & 1587 & 1578 \\
4 & 1560 & 1610 & 1596 \\
5 & 2120 & 1725 & 1570 \\
6 & 1680 & 1611 & 1576 \\
7 & 1696 & 1596 & 1558 \\
8 & 1584 & 1577 & 1566 \\
9 & 1576 & 1586 & 1574 \\
10 & 1612 & 1579 & 1564 \\
\hline
\end{tabular}

\section{Conclusion}

Aiming at the low efficiency of the automated storage and retrieval system, the scheme of optimizing the cargo space management and job scheduling based on the expert system was put forward in this paper. The overall structure of the expert system was designed, the knowledge base based on the production rules was established and the reasoning process based on the forward reasoning method was designed. Experiments show that the application of the expert system for cargo space management and job scheduling optimization can reduce the running time of stacking machine and can improve the efficiency of automated storage and retrieval system effectively. 


\section{Acknowledgements}

Fund projects: The research topic of teaching reform in Jiangxi higher schools (JXJG-16-27-11), The research topic of teaching reform in Nanchang Institute of Technology(NGJG-2015-34).

This work was supported in part by the National Science Foundation of China (51765042, 61463031, 61662044, 61773051), Jiangxi Provincial Department of Science and Technology (20142BBE50037, 20151BBE50050), and Jiangxi Provincial Department of Education (GJJ151236, JXYJG-2017-020, GJJ151234, GJJ161225).

\section{References}

[1] Guan Hong.Research on Design Method of Overall Plan for Automatic Storage[D].Xi'an:Northwestern Polytechnical University, 2011.

[2] Tian Guohui, Liu Changyou, Lin Jiaheng,et al.The Research and Progress of Problems That Optimized Scheduling in AutomatedStorage[J].Journal of Shandong University of Technology, 2001, 31(1): 12-17.

[3] Li Meijuan,ChenXuebo, Liu Chenqi.TheResearch of Access Equipment on Optimal Scheduling Method for Automatic Storage[J]. Computer Engineering and Design,2006, 27(2): 181-183.

[4] Chang Faliang, Liu Zengxiao,XinZheng, etc.Research on Optimization of Pick - up Operation Path in Automatic Storage[J]. Theory and Practice of System Engineering, 2007, 5(2): 139-143.

[5] Liu Sainan, KeYinlin, Li Jiangxiong, et al.Research on Optimization of Automated Warehouse System Based on Dispatching Strategy[J].Computer integrated manufacturing system, 2006, 12(9): 1 438-1 443.

[6] Siegel P H. Terahertz technology[J]. IEEE Transactionson Microwave Theory and Techniques, 2002, 50(3): $910-928$.

[7] Hu Yahong, Chen huanyu. Travel time analysis of a newautomated storage and retrieval system [J]. Computers \& Operations Research, 2005, 32(6): 1 515-1 518.

[8] Tian Guohui, Zhang Pan. Research on Optimization of Fixed Shelf Selection Based on Hybrid Genetic Algorithm[J]. Journal of Mechanical Engineering, 2004,40 (2): 141-144.

[9] Liu Xiaozeng, Feng Zhanying, Wu Jian, et al. Optimization algorithm of job path for picking automatic warehouse stacking machine [J]. Lifting and Transportation Machinery, 2006,20 (8): 49-51.

[10]Cheng Weiliang. Generalized expert system and its application [M]. Beijing: Beijing Institute of Technology Press, 2005. 